\title{
System integration of sensors for fluorescence based lab on a chip systems
}

\author{
Andreas Witte, Robert Schmitt \\ Laboratory for Machine Tools and Production Engineering (WZL) of RWTH Aachen University \\ Steinbachstrasse 19, 52074 Aachen
}

\section{INTRODUCTION}

Lab on a chip (LOC) devices are small microfluidic systems which can partly replace big laboratories for clinical diagnosis [1]. Especially in applications where rapid analysis is important, LOCs can increase the patient's ability to heal. They have a lot of advantages over standard laboratories, such as fast processing and high portability [2].

Besides the chip itself, all instrumentation which is necessary to control the fluids and the analysis is a very fundamental part of the whole system. This is why the system integration of fluidic components and sensors have to be a part of the development process of a lab on a chip device.

This article will describe the integration of fiber optical sensors in an analysis module for lab in a chip systems. It expands further on the specific challenges of the integration process, which is for example a definite and repeatable positioning of the chip relative to the sensors.

\section{INSTRUMENTATION AND SYSTEM DESIGN}

The entire system consists of only a few core components, which is the microfluidic chip, the optical fluorescence detection device and a fluid handling system. All these components are connected to an analysis module which integrates the sensors, the fluid tubes and positions the chip.

The chip, that is used the analysis module, consists of different coats and is manufactured in a lithographic process. On a base silicon or glass substrate a photoresist is superimposed, which can carry the microfluidic structures. The height and width of these structures are in the order of magnitude of some tens of microns. Finally, the photoresist is covered with a plate to assure impermeability.

Additionally, plasma assisted surface functionalization can define the position of spots for a fluorescence detection. This is realized by electrodes on the chip. An electrical potential between these electrodes causes a plasma reaction, which leads to specific surface modifications. [3], [4]

An essential part of the instrumentation for a microfluidic device is the fluid handling system. A contamination of the specimen with these of other patients would lead to wrong results and therefore to an incorrect diagnosis. To avoid this, a liquid handling strategy including a specific component configuration is needed. In this configuration the specimen only has contact with defined tubes and valves for example. The liquid handling system consists of a syringe pump and various valves, which can control the liquid flow (Fig. 1, Fig. 2).

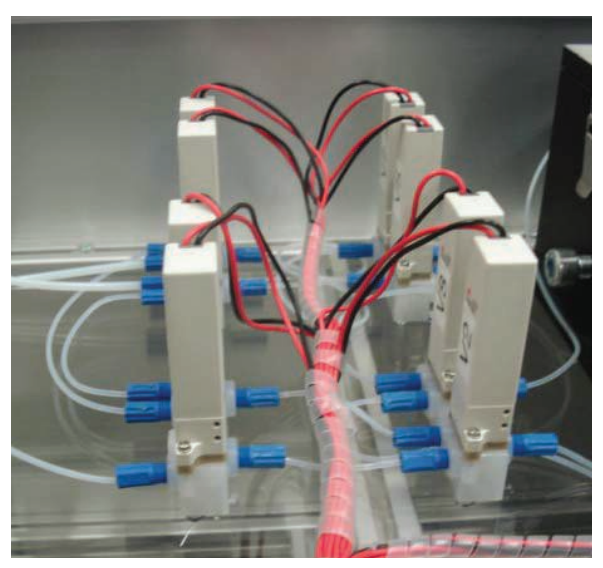

Fig. 1: Valves for liquid handling

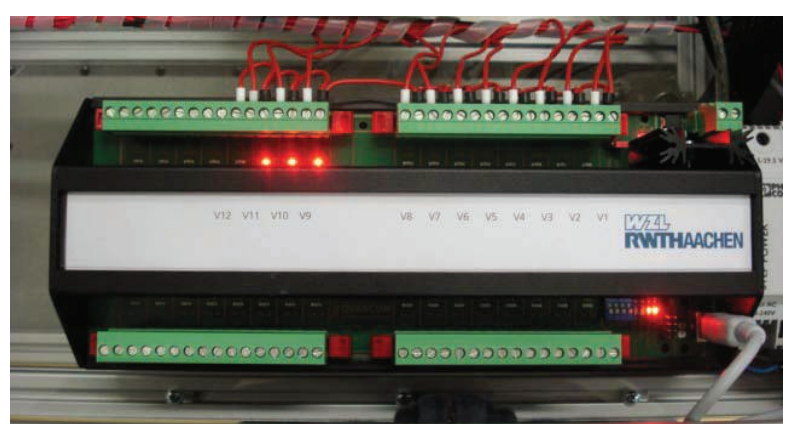

Fig. 2: Control unit for liquid handling 


\section{FLUORESCENCE SPECTROSCOPY}

For a quantitative fluorescence based detection of markers in a specimen a time resolved detection system is used. Although the instrumentation is a lot more complex, some of the molecular information from a fluorescence process is only available with time resolved systems. [5] In general the fluorescence intensity can be calculated with [6]:

$$
\frac{I_{f}}{I_{0}}=\Phi_{f}\left(1-10^{\varepsilon C L}\right)
$$

where $I_{0}$ is the intensity of the light source, $I_{f}$ the intensity of fluorescence, $\Phi_{f}$ the quantum efficiency and $\varepsilon \mathrm{CL}$ the product of molar absorptivity, molar concentration and optical path length. A usual time of life for this phenomenon is in the order of magnitude of $10 \mathrm{~ns}$ and has an exponential characteristic [6]:

$$
I(t)=I_{0} e^{-t / \tau_{f}^{0}}
$$

where $T_{f}^{0}$ is the fluorescence lifetime. The equations above already show some of the requirements for the optical and electrical instrumentation. The losses of intensity of both the excitation and the fluorescence channel should be held as low as possible to achieve a high signal to noise level. Moreover, the light source and the detectors have to be fast enough to record the measurement data with a sufficient resolution for a time based processing.

In the current experimental setup the detection is realized by photomultipliers with a high resolution and sensitivity. As a light source a laser is used for the excitation and is optically decoupled from the detector using several filters.

\section{SENSOR DESIGN AND ITS REQUIREMENTS FOR A SYSTEM INTEGRATION}

The quality of the measurements and therefore the quality of the results for a diagnosis, is depending on the fluorescence system. But the instrumentation itself is only one part to define the quality. The second part is the sensor and the method of its integration.

There are several alternatives existing for the connection of the instrumentation to the chip [7-9]. Some approaches focus on an integration of fibers or waveguide structures in the chip [10]. A very flexible method for cost sensitive applications is the use of fibers without an integration in the chip. This allows the fibers to be in the non-disposable part of the system, while the sensitivity of the whole device is comparable to systems with an on-chip integration. Moreover, fibers are brought close to the detection spots of the chip in the current experimental setup to reduce fluorescence signal losses.

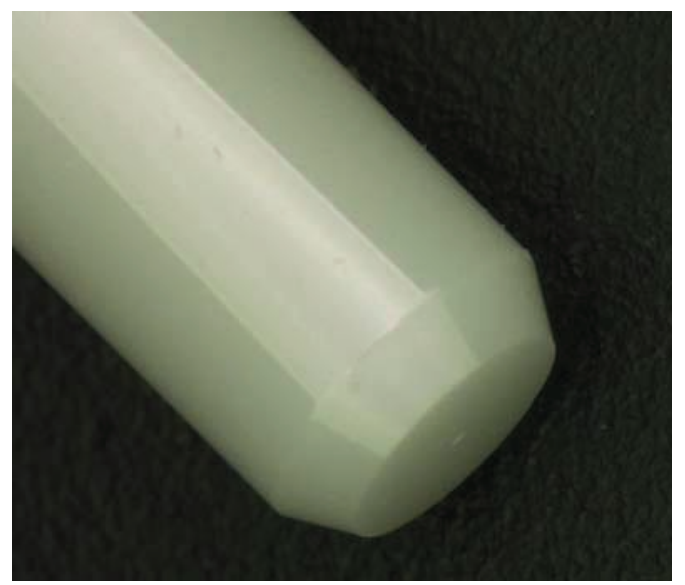

Fig. 3: Sensor head 


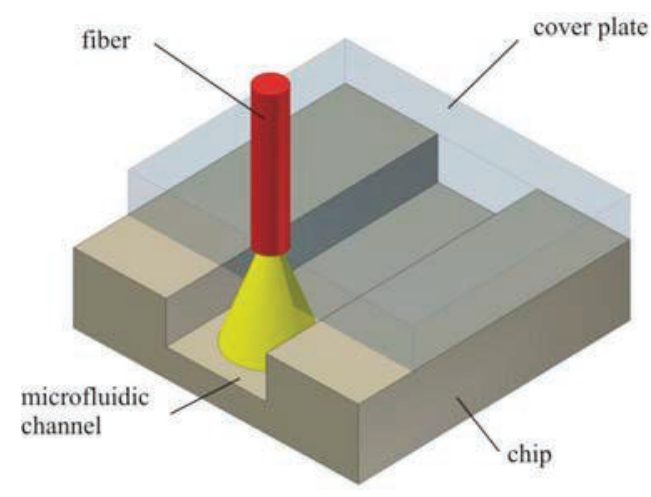

Fig. 4: Fiber and detection spot

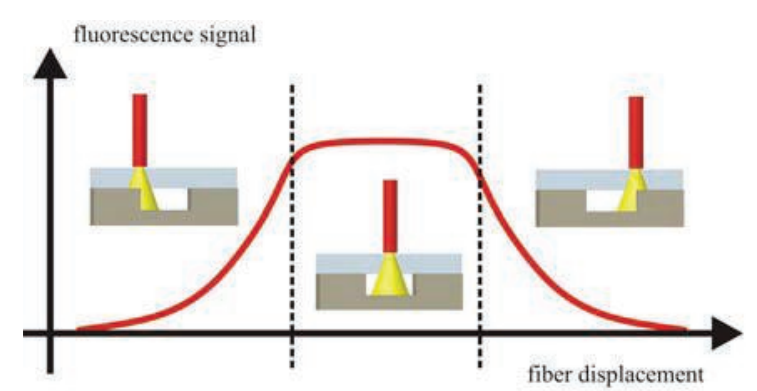

Fig. 5: Effect of fiber displacement

In various projects at least two different fibers are used for the excitation and the fluorescence signal, so that an interference between them can be avoided [11]. To consider the miniaturization and cost efficiency requirements, there is only one fiber for the excitation and emission used in our experimental setup.

One challenge of the optical and mechanical system is the high usability. The position of the chip relative to the fibers has to be accurate to assure reproducible measurement results. However, the user of the system should not be forced to readjust the position of the chip in its fixture. These complementary requirements have to be considered in the system design.

To increase the cost efficiency, the sensors have to be designed without any further beam-shaping optical elements. As sensor a bare fiber end in a FC-ferrule (Fig. 3) can be used and mounted in the fixture without displacement, because of the narrow tolerances of the ferrule. Nevertheless, this also means that the excitation beam has a divergent propagation, as well as the emission acceptance cone is defined by the numerical aperture of the fiber (Fig. 4). In the current project the half acceptance angle is $\theta \approx 8.47^{\circ}$. As a result, the base circle diameter of the beam is in the order of magnitude of some tens of microns, the fiber being close to the detection spot. According to that fact, a displacement of the fiber relative to the chip can cause significant signal losses because the excitation and emission beams are not matching the microfluidic channel. This effect is shown in Fig. 5 qualitatively. To avoid signal losses and to assure reproducible measurements a precision positioning device was developed and characterized.

\section{EXPERIMENTAL SETUP}

The precision positioning device, which was developed, consists of two parts (Fig. 6), a base and a cover plate. To change the microfluidic chip, which is placed on a platform in the base, the cover plate can be applied to the base and removed from it.

To connect the tubes for the liquid handling to the chip, fittings are used. Therefore, tapped holes are in the cover plate, where the fittings can be applied. To connect the sensors to the cover plate, bores with a cylindrical interference fit are used. For mounting the sensors, it is therefore necessary to heat the bores, so that the thermal expansion can be used for the process. After the plate is cooled down to room temperature, the ferrules are fixed in a well-defined position in the cover plate.

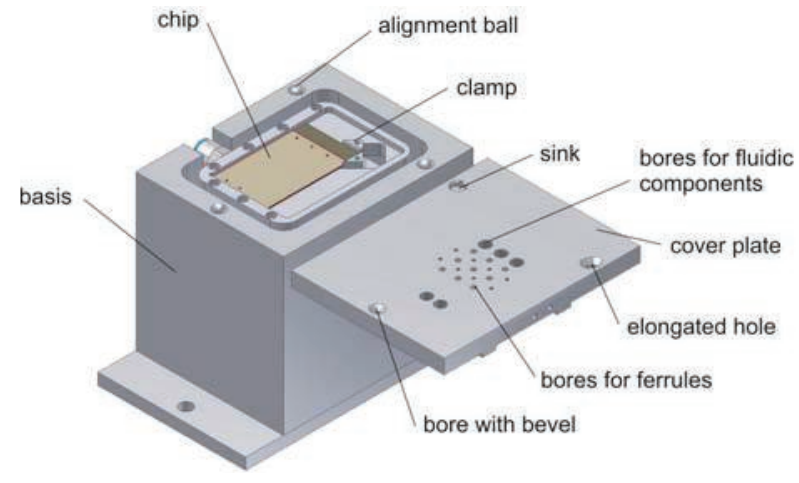

Fig. 6: Positioning stage

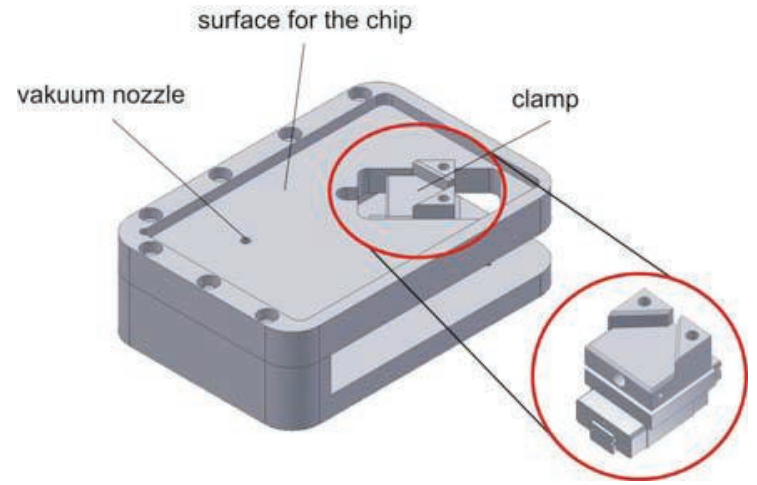

Fig. 7: Fixture for the LOC 


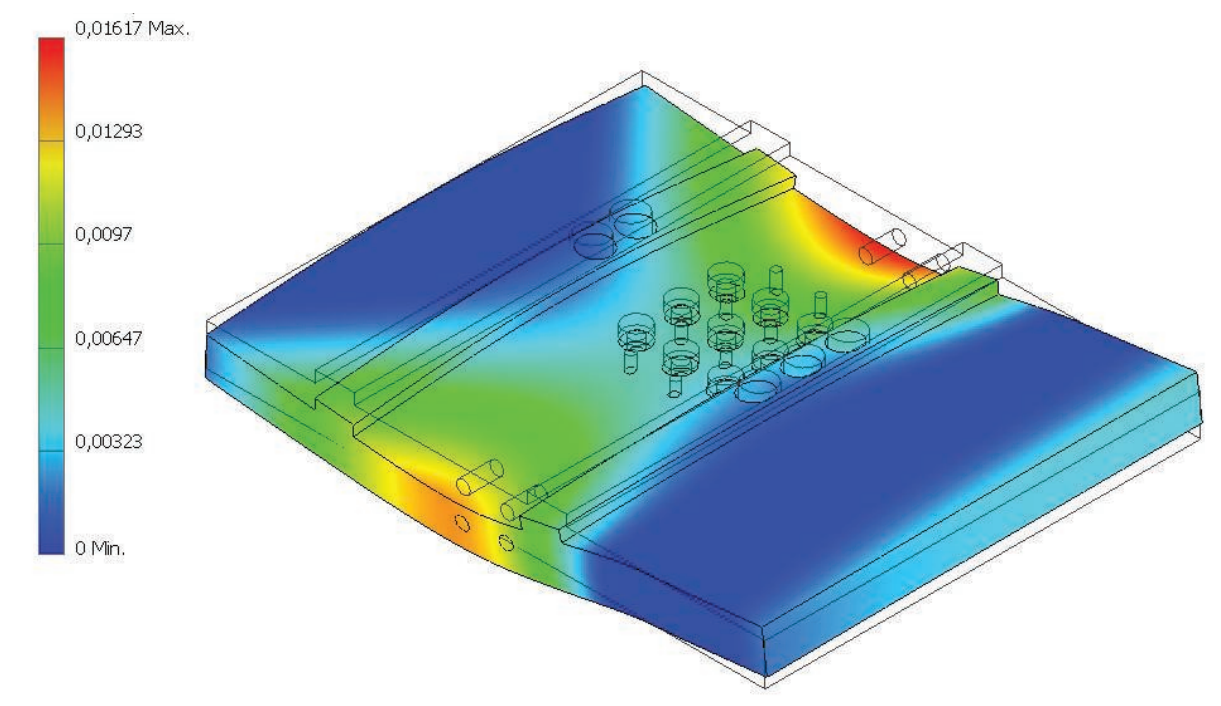

Fig. 8: Results of the FEM analysis of the cover plate (displacement in $\mathrm{mm}$ )

There are two possibilities where a misalignment could effect the fluorescence measurements. The first one is a displacement of the cover plate with the integrated sensors relative to the base. This would automatically cause a displacement of the fibers relative to the chip which leads to significant fluorescence signal losses. The second possibility is a misalignment of the chip relative to the base, which equally causes sensor positions that can no longer match the detection spots.

To assure an aligned position of the cover plate relative to the base, it is necessary to use aligning structures. The use of aligning pins is an alternative, which is not applicable in the case at hand. The cover plate is applied to the base and removed every time the chip has to be changed. This would cause a high mechanical stress for the alignment pins and bores which would lead to damages and less precise positioning [12], [13]. The solution which is used in the present case is a combination of bores in the cover plate and in the base with balls as aligning structures in between these two elements.

In the base, three bores with bevels are used for a well-defined position of the balls. The corresponding structures in the cover plate need a more complex geometry. One structure is as well a bore with bevel to fix all translational dimensions of freedom. An elongated bore and a sink are used to fix the cover plate in all rotatory dimensions of freedom (Fig. 6).

This aligning concept assures a high repeat accuracy of the position of the cover plate and avoids a statically indeterminate system. Furthermore, thermal expansions during the measurement process, which are caused for example through the user or the specimen, can be balanced.

In contrast, the displacement of the chip relative to the base is minimized with another structural concept (Fig. 7). The fixture consists of two layers. On the lower layer a clamp is installed with a combination of a linear and a radial bearing to allow a two-dimensional movement of the clamp. On the upper layer, the surface for carrying the chip is located. Its edges act as passive alignment structures for the chip, which is fixed by a vacuum nozzle in the middle of the surface. After applying the chip, it is moved to its reference position with the clamp, which is on the lower level fixed with a spring. To avoid displacements, the clamping jaws have an angle of less then $90^{\circ}$.

\section{DISCUSSION}

To assure high repeat accuracies, it is important to already evaluate the mechanical structures in the design process. Therefore, a finite elements analysis (FEM) was performed with the cover plate. The catches, which fix the cover plate on the base, are producing a defined force and therefore a bending of the plate. In the worst case the catches can apply a maximum force of $150 \mathrm{~N}$ each, so this is considered as the basis for the calculation. Fig. 8 shows the result of the FEM analysis. The maximum displacement of any sensor related part of the cover plate has been calculated to 8.8 microns and is accordingly tolerable. 


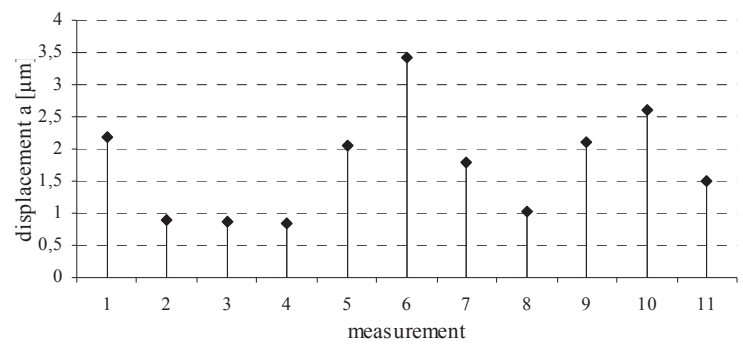

Fig. 9: Repeat accuracy of cover plate relative to base

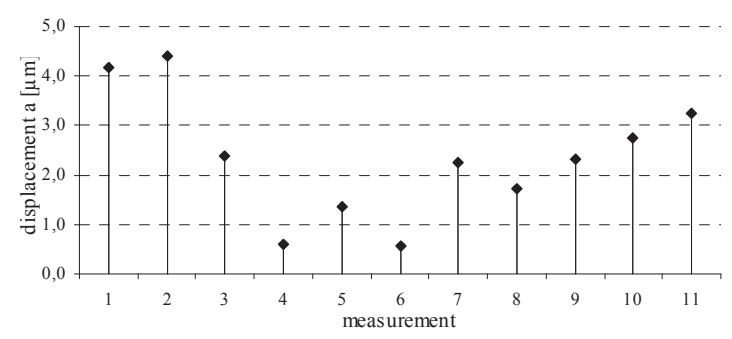

Fig. 10: Repeat accuracy of chip relative to base

To review the whole concept, repeat accuracies of the chip and the cover plate relative to the base have to be evaluated. It is estimated that the repeat accuracies for all parts is in the order of magnitude of some microns. Consequently, the measurement setup and concept has to meet the requirements in the points of measuring uncertainty. For this reason, a Werth UA400 multi-sensor coordinate measuring machine was used for the evaluation, which guarantees a measuring uncertainty of less than one micron. The measurement of the displacement of the cover plate relative to the base can be performed with a tactile measuring method. For the repeat accuracy, the cover plate is applied to the base and several times removed and reapplied. After every application the position of the plate is measured. The results of this measurement are shown in Fig. 9. The maximum deviation from the arithmetic mean does not exceed 3.5 microns for eleven repetitions.

For the measurement of the displacement of the chip relative to the base an image processing measurement is required. A tactile measurement method is not applicable because the chip has a height of only several hundred microns and its edges do not allow a touch of the sensor head. In contrast, the measurement procedure is similar to the procedure described above. Hence, the chip is applied to the base and removed from it several times, measuring the deviation of its position every iteration. The results (Fig. 10) show, that the deviation of the mean does not exceed 4.5 microns in this case.

\section{CONCLUSION}

The sensor and system integration is an essential part in the design process for LOC devices. Particularly, the structural design has to be evaluated in an early stage of this process. The results show, that a precision positing device for biomedical applications can be realized with low mechanical complexity. The concept of using aligning balls as an alternative to aligning pins, assure high repeat accuracies on low manufacturing costs. Likewise, the clamping device reduces misalignments of the chip so that additionally the influence on the fluorescence measurement is diminished.

The concepts developed and evaluated in this project, can be transferred into applications outside the biomedical engineering. The aligning concept and structural approach is for example suitable for microassembly and measurement applications.

Though, it has to be considered that the design process and structural preparation are, beside the ultra precision machining of bearing surfaces, cost factors that should not be disregarded.

\section{ACKNOWLEDGMENT}

The authors would like to acknowledge financial support by the Exploratory Research Space of RWTH Aachen University within the research project "FIND-IT" (OPBo14). Additionally, the authors thank the Institute of Biology VII, the Department of Experimental Medicine and Immunotherapy at the Chair of Applied Medical Engineering, the Institute of Materials in Electrical Engineering (Chair 1) and the Chair for Laser Technology of RWTH Aachen University for their cooperation during the project.

\section{REFERENCES}

[1] D. Li, "Microfluidic Lab-on-a-Chip Devices for Biomedical Applications", NATO Science for Peace and Security Series A: Chemistry and Biology, vol. 0, 2010, pp. 377-397. 
[2] D. Mark, S. Haeberle, G. Roth, F. von Stetten, R. Zengerle, "Microfluidic lab-on-a-chip platforms: requirements, characteristics and applications", Chem. Soc. Rev., vol. 39, 2010, pp. 1153-1182.

[3] B. Mitra, C. Wilson, L. Que, P. Selvaganapathy, Y. Gianchandani, "Microfluidic discharge-based optical sources for detection of biochemicals", Lab Chip, vol. 6, 2006, pp. 60-65.

[4] U. Kogelschatz, "Applications of microplasmas and microreactor technology," Contrib. Plasma Phys. 47, Vol. 1-2, 2007, pp. 81-88.

[5] J. Lakowicz, Principles of fluorescence spectroscopy, 3er ed. Berlin: Springer, 2006, pp.14-15.

[6] Sharma, Ashutosh, and Stephen Schulman. Introduction to Fluorescence Spectroscopy. John Wiley \& Sons, Inc., 1999, pp. 22-23, 101-104

[7] L. Novak, P. Neuzil, J. Pipper, Y. Zhang, S. Lee, "An integrated fluorescence detection system for lab-on-a-chip applications", Lab Chip, vol. 7, 2007, pp. 27-29.

[8] R. Mazurczyk, J. Vieillard, A. Bouchard, B. Hannes, S. Krawczyka, "A novel concept of the integrated fluorescence detection system and its application in a lab-on-a-chip microdevice", Sensors and Actuators B, vol. 118, 2006, pp. 11-19.

[9] O. Wolfbeis, "Fiber-Optic chemical sensors and biosensors", Anal. Chem., vol. 76, 2004, pp. 32693284.

[10] B. Bilenberg, T. Nielsen, B. Clausen, A. Kristensen, "PMMA to SU-8 bonding for polymer", Journal of Micromechanics and Microengineering, vol. 14, 2004, pp. $814-818$.

[11] L. Marcua, J. Jod, Q. Fange, T. Papaioannouf, T. Reilc, J.-H. Qiaob, J. Bakerc, J. Freischlagg, M. Fishbeinb, "Detection of rupture-prone atherosclerotic plaques by time-resolved laser induced fluorescence spectroscopy", Atherosclerosis, vol. 204, 2009, pp. 156-164.

[12] D. Muhs, H. Wittel, M. Becker, D. Jannasch, J. Voßiek, Roloff/Matek Maschinenelemente, 17th ed. Wiesbaden: Vieweg, 2003, pp. 257-259.

[13] G. Niemann, H. Winter, B.-R. Höhn, Maschinenelemente, vol. 1, 4th ed. Berlin: Springer, 2005, pp. 466-467. 